11.4

\title{
Обнаружение хаотических радиоимпульсов с помощью ансамбля детекторов огибающих
}

\author{
() Ю.В. Андреев
}

Московский физико-технический институт (Национальный исследовательский университет), Долгопрудный, Московская обл., Россия

Институт радиотехники и электроники им. В.А. Котельникова РАН, Москва, Россия

E-mail: andreev.iuv@mipt.ru

Поступило в Редакцию 31 августа 2020 г.

В окончательной редакции 21 сентября 2020 г.

Принято к публикации 21 сентября 2020 г.

Исследуется возможность обнаружения сверхширокополосных хаотических радиоимпульсов с помощью ансамбля некогерентных приемников на основе детекторов огибающей. Получено аналитическое выражение для вероятности ошибки приема как функции отношения сигнал/шум на входе детектора огибающей. Показано, что чувствительность детектирования радиоимпульсов (по параметру отношения сигнал/шум) улучшается пропорционально количеству детекторов.

Ключевые слова: детектор огибающей, динамический хаос, сверхширокополосный радиоимпульс, приемник.

DOI: 10.21883/PJTF.2021.01.50454.18534

В настоящее время наблюдается интерес к многоэлементным системам передачи и приема информации $[1,2]$. Зачастую они представляют собой многоканальные системы связи со специальным формированием диаграмм направленности, которые позволяют существенно увеличить агрегированную скорость передачи [3]. Многоэлементные передача и прием исследуются и в системах связи на сверхширокополосных сигналах [4], в том числе и с хаотическими несущими [5].

Однако многоэлементные системы связи можно использовать не для увеличения объема передачи, а для увеличения ее дальности. Эта проблема является довольно острой в области беспроводной сверхширокополосной прямохаотической радиосвязи [6,7]. В прямохаотических системах несущим сигналом являются широкополосные или сверхширокополосные хаотические колебания, формируемые непосредственно в полосе частот канала связи; информация кодируется потоком хаотических радиоимпульсов, а прием осуществляется также непосредственно в полосе частот канала с помощью энергетического приемника (детектора огибающей) [4]. Из-за ограничений на спектральную плотность излучения [8] передающей системы дальность действия ограничена десятками метров. Использование многоэлементных систем как на передачу, так и на прием может существенно увеличить дальность действия сверхширокополосных прямохаотических систем.

Применение многоэлементных систем на передающей стороне было исследовано нами ранее и показало [9-11], что за счет коллективного излучения сверхширокополосных хаотических радиоимпульсов ансамблем независимых хаотических излучателей мощность передачи возрастает пропорционально числу излучателей, а ввиду некогерентности волн, приходящих в точку приема от разных излучателей, обужения диаграммы направленности не происходит.

Цель настоящей работы - исследование возможностей многоэлементного приемника беспроводной сверхширокополосной прямохаотической радиосвязи.

Рассмотрим многоэлементную систему обнаружения сверхширокополосных хаотических радиоимпульсов, представленную ансамблем $M$ детекторов огибающей (рис. 1). Примем, что детекторы являются квадратичными, т. е. амплитуда импульса огибающей на выходе детектора пропорциональна энергии (мощности) радиоимпульса на входе. Также примем, что прием ведется в дальней зоне и что фронт радиоволны передатчика достигает антенн всех приемников одновременно. Канал связи будем считать статическим, шум в канале гауссовым; многолучевого распространения нет.

Рассмотрим преобразования сигнала в детекторе огибающей (рис. 2).

Обозначим сигнал на входе детектора $X(t)$. Задача заключается в обнаружении радиоимпульса на известном временно́м интервале; обозначим его $[0, T]$, где $T$ - длина радиоимпульса. Для ортогональной системы сигналов требуется различать два следующих случая:

$$
X_{1}(t)=s(t)+\xi(t), t \in[0, T]-\text { передача ,1“ }
$$

(наличие радиоимпульса),

$$
\begin{gathered}
X_{0}(t)=\xi(t)-\text { передача „0“ } \\
\text { (отсутствие импульса), }
\end{gathered}
$$

где $s(t)$ - несущий хаотический сигнал, формируемый детерминированной динамической системой, спектр $S(f)$ которого ограничен полосой $\left[f_{l}, f_{u}\right] ; \xi(t)-$ 


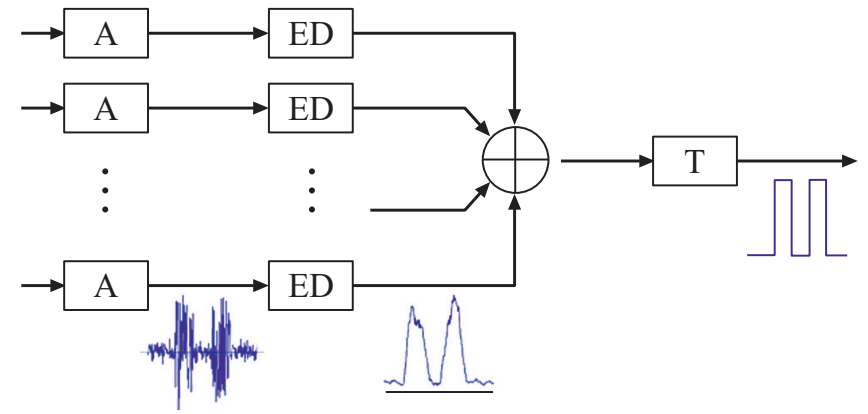

Рис. 1. Приемный ансамбль. А - антенна, ED - детектор огибающей, Т - пороговое устройство.

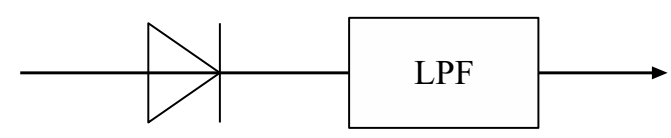

Рис. 2. Блок-схема детектора огибающей.

шум приемника, приведенный ко входу детектора. В рамках данной модели будем представлять его „белым“ шумом, ограниченным той же частотной полосой $\left[f_{l}, f_{u}\right]$.

Рассмотрим модель детектора огибающей, состоящего из квадратичного диода и фильтра низких частот (LPF) (рис. 2). При прохождении сигнала $X(t)$ через детектор на выходе диода получаем сигнал $X^{2}(t)$ исходной частотной полосы. Этот сигнал, соответствующий мгновенной мощности принятого сигнала, поступает на вход LPF. Так как фильтр низких частот фактически является интегратором, на выходе его получаем сигнал, пропорциональный энергии входного сигнала на некотором предшествующем временно́м интервале (окне) $T_{w}$. Если постоянная времени фильтра согласована с длиной радиоимпульса $T$, а момент взятия отсчета на выходе детектора соответствует моменту, когда окно фильтра совпадает с интервалом радиоимпульса $[0, T]$, то отсчет сигнала на выходе детектора огибающей $X_{D}(T)$ равен энергии радиоимпульса на интервале $[0, T]$, т.е. $E_{1}$ при передаче символа „1“ или $E_{0}$ при передаче символа „0“ (здесь и далее подстрочные индексы 1 и 0 в обозначениях переменных и функций относятся соответственно к случаям наличия или отсутствия импульса).

Раскроем выражение для $E_{1}$

$$
\begin{aligned}
E_{1}= & \int_{0}^{T}(s(t)+\xi(t))^{2} d t=\int_{0}^{T} s^{2}(t) d t+\int_{0}^{T} \xi^{2}(t) d t \\
& +2 \int_{0}^{T} s(t) \xi(t) d t .
\end{aligned}
$$

Хаотический сигнал $s(t)$ и гауссова помеха $\xi(t)$ являются некоррелированными величинами с нулевым средним, поэтому кросскорреляционным членом в (2) можно пренебречь.
Для дальнейшего анализа удобно перейти от непрерывного представления сигналов к дискретному, тогда

$$
\begin{gathered}
E_{1}=\sum_{i=1}^{n} s_{i}^{2}+\sum_{i=1}^{n} \xi_{i}^{2}, \\
E_{0}=\sum_{i=1}^{n} \xi_{i}^{2} .
\end{gathered}
$$

Здесь $s_{i}$ и $\xi_{i}$ - независимые отсчеты на интервале $[0, T]$. Поскольку соответствующие непрерывные сигналы занимают полосу частот $f \in\left[f_{l}, f_{u}\right]$, согласно теореме Котельникова, отсчеты следует брать через интервалы времени $\Delta t=1 / 2 \Delta f=\frac{1}{2\left(f_{u}-f_{l}\right)}$. Тогда $n=\frac{T}{\Delta t}=2 T \Delta f=2 B$, где $B=\Delta f \Delta T-$ база сигнала.

Энергия $E_{0}$ в выражении (4) есть сумма квадратов отсчетов случайной помехи. Поскольку здесь рассматривается случай гауссова шума (AWGN) на входе детектора, отсчеты помехи имеют нормальное распределение $N\left(0, \sigma_{\text {noise }}^{2}\right)$. Соответственно сумма квадратов таких отсчетов описывается распределением $\chi^{2}$ с $n$ степенями свободы с моментами $E[\chi]=n \sigma_{\text {noise }}^{2}, D[\chi]=2 n \sigma_{\text {noise }}^{2}$, которое в свою очередь, согласно центральной предельной теореме [12], при $n \rightarrow \infty$ стремится к нормальному распределению $N\left(n \sigma_{\text {noise }}^{2}, 2 n \sigma_{\text {noise }}^{4}\right)$. В практических приложениях прямохаотической связи условие $n \gg 1$ уверенно выполняется [6,7], поэтому $E_{0}$ можно с хорошей точностью аппроксимировать выражением

$$
E_{0}=\sum_{i=1}^{n} \xi_{i}^{2}=n \sigma_{\text {noise }}^{2}+\zeta_{\text {noise }},
$$

где $\xi_{\text {noise }}$ - случайная величина, описываемая нормальным распределением $N\left(0,2 n \sigma_{\text {noise }}^{4}\right)$.

Оценки $E_{1}$ определяются используемой моделью хаотического сигнала. В работах $[13,14]$ были проанализированы различные типы хаотических сигналов и соответствующие модели таких сигналов, а также путем численного моделирования были выполнены оценки характеристик систем связи для нескольких типов хаотической несущей. В частности, было показано, что „фазовый“ хаос дает лучшие показатели системы передачи по параметру вероятности ошибки (BER), чем „амплитудный“ хаос, так как вариации энергии радиоимпульсов с фазово-хаотическим заполнением незначительны, в то время как для амплитудно-хаотического сигнала такие вариации существенны, что представляет собой неизбежную дополнительную помеху.

В настоящей работе рассмотрим вариант „амплитудного“ хаоса как более общий. При достаточно широкой полосе $\left[f_{l}, f_{u}\right]$ дискретные отсчеты хаотического сигнала $s_{i}$ с ровной спектральной характеристикой, взятые с тем же шагом $\Delta t$, с хорошей точностью описываются нормальным распределением с нулевым средним и дисперсией $D=\sigma_{\text {chaos }}^{2}$.

Тогда в выражении (3) для $E_{1}$ значения энергии радиоимпульса $\Sigma s_{i}^{2}$ тоже описываются распределением 


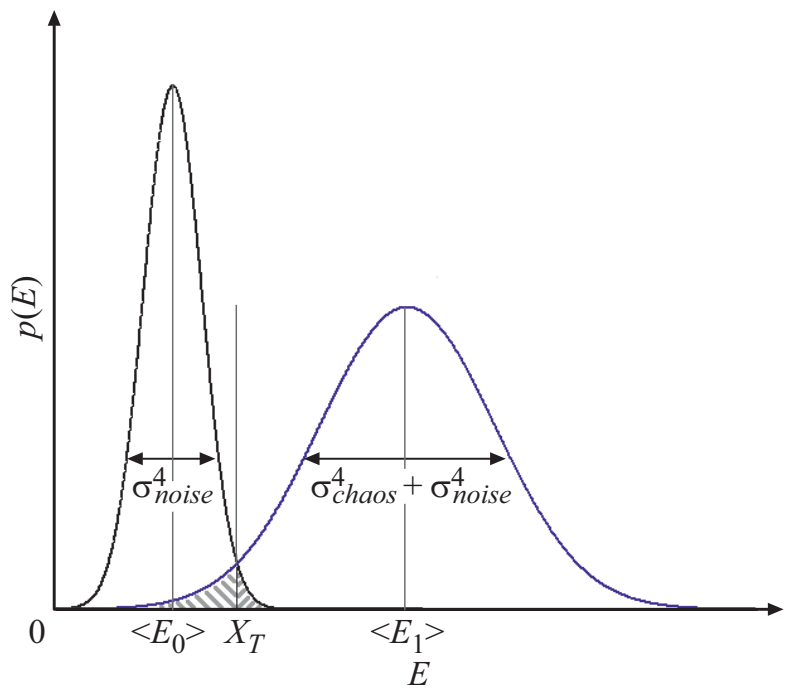

Рис. 3. Функции распределения энергии принятого сигнала для символов „,““ и „„“.

$\chi^{2}$, которое, так же как в выражении для $E_{0}$, можно аппроксимировать нормальным распределением. В этом случае

$$
\begin{aligned}
E_{1}= & \sum_{i=1}^{n} s_{i}^{2}+\sum_{i=1}^{n} \xi_{i}^{2}=n\left(\sigma_{\text {chaos }}^{2}+\sigma_{\text {noise }}^{2}\right) \\
& +\xi_{\text {chaos }}+\xi_{\text {noise }}=\xi_{\Sigma},
\end{aligned}
$$

где $\xi_{\Sigma}-$ отсчеты с нормальным распределением $N\left(n\left(\sigma_{\text {chaos }}^{2}+\sigma_{\text {noise }}^{2}\right), 2 n\left(\sigma_{\text {chaos }}^{4}+\sigma_{\text {noise }}^{4}\right)\right)$.

Таким образом, распределения отсчетов описываются следующими функциями:

$$
\begin{gathered}
w_{0}(x)=\frac{1}{\sigma_{\text {noise }}^{2} \sqrt{4 \pi n}} e^{-\frac{\left(x-\overline{E_{0}}\right)^{2}}{4 n \sigma_{\text {noise }}^{4}},} \\
w_{1}(x)=\frac{1}{\left(\sigma_{\text {chaos }}^{2}+\sigma_{\text {noise }}^{2}\right) \sqrt{4 \pi n}} e^{-\frac{\left(x-\overline{E_{1}}\right)^{2}}{4 n\left(\sigma_{\text {chaos }}^{4}+\sigma_{\text {noise }}^{4}\right)}},
\end{gathered}
$$

где $\overline{E_{0}}=n \sigma_{\text {noise }}^{2}, \overline{E_{1}}=n\left(\sigma_{\text {chaos }}^{2}+\sigma_{\text {noise }}^{2}\right)-$ средняя энергия принятого сигнала на интервале $[0, T]$ соответственно при отсутствии и наличии радиоимпульса (рис. 3 ).

Для различения принятого символа введем порог $X_{T}$ : если сигнал $X_{D}$ на выходе ниже порога, т. е. $X_{D}(T)<X_{T}$, то принят символ „о“, в противном случае принят символ ,1“.

Вероятность ошибки $P_{\text {err }}$ складывается из вероятностей ложного обнаружения для каждого из символов (соответствует заштрихованной области на рис. 3). С учетом вероятности передачи символов $p(0)$ и $p(1)$ получаем

$$
\begin{gathered}
P_{\text {err } r}=P_{\text {err }, 0}+P_{\text {err }, 1}=p(0) \int_{X_{T}}^{\infty} w_{0}(x) d x \\
+p(1) \int_{-\infty}^{X_{T}} w_{1}(x) d x .
\end{gathered}
$$

В случае равновероятно передаваемых символов $p(0)=p(1)=0.5$ порог $X_{T}$ определяется уравнением $w_{1}\left(X_{T}\right)=w_{0}\left(X_{T}\right)$, отсюда

$$
\begin{aligned}
P_{\text {err }}= & \frac{1}{2}-\frac{1}{2} \Phi\left(\frac{X_{T}-\overline{E_{0}}}{\sqrt{2 n} \sigma_{\text {noise }}^{2}}\right) \\
& +\frac{1}{2} \Phi\left(\frac{X_{T}-\overline{E_{1}}}{\sqrt{2 n\left(\sigma_{\text {chaos }}^{4}+\sigma_{\text {noise }}^{4}\right)}}\right),
\end{aligned}
$$

где $\Phi(x)=\frac{1}{\sqrt{2 \pi}} \int_{-\infty}^{X} e^{-t^{2} / 2} d t-$ кумулятивная функция нормального распределения.

Выше были описаны преобразования сигнала в одном канале обнаружителя сверхширокополосных хаотических радиоимпульсов. Рассмотрим теперь, что произойдет, если суммировать выходные сигналы детекторов огибающей перед решающим (пороговым) устройством.

При наличии радиоимпульса на выходе $i$-го детектора имеется единичный отсчет вида

$$
E_{1 i}=a+\xi_{i}, \quad i=1, \ldots, M
$$

где $a=n\left(\sigma_{\text {chaos }}^{2}+\sigma_{\text {noise }}^{2}\right)$, а помеха $\xi_{i}=\xi_{\text {chaos }, i}+\zeta_{\text {noise }, i}$ имеет нормальное распределение с нулевым математическим ожиданием и дисперсией $D \xi_{i}=2 n\left(\sigma_{\text {chaos }}^{4}+\sigma_{\text {noise }}^{4}\right)$.

Отсчеты случайной помехи $\xi_{i}$ в каждом детекторе являются независимыми, так как физически они определяются тепловыми шумами отдельных приемных каналов, которые технически представляют собой несвязанные устройства. В то же время, поскольку все детекторы огибающей конструктивно одинаковы, можно считать, что шумы отдельных каналов подчиняются одной и той же статистике.

Суммирование отсчетов (11) дает сигнал на входе порогового устройства

$$
E=M a+\sum_{i=1}^{M} \xi_{i}=b+\eta
$$

где $b=M a$ представляет собой полезный сигнал, а случайная величина $\eta-$ суммарную помеху. Тогда отношение сигнал/шум на входе порогового устройства 
составляет

$$
\begin{gathered}
\rho=\frac{P_{s}}{P_{n}}=\frac{b^{2}}{D \eta}=\frac{M^{2} a^{2}}{D\left(\Sigma \xi_{i}\right)}=\frac{M^{2} a^{2}}{\Sigma D \xi_{i}}=\frac{M^{2} a^{2}}{M D \xi_{i}} \\
=M \frac{a^{2}}{D \xi_{i}}=M \rho_{c h} .
\end{gathered}
$$

где $\rho_{c h}=\frac{a^{2}}{D \xi_{i}}-$ отношение сигнал/шум на выходе каждого приемного канала.

Итак, после сумматора отношение сигнал/шум увеличивается в $M$ раз. Это связано с тем, что при суммировании мощность полезного сигнала увеличивается в $M^{2}$ раз (амплитуда в $M$ раз), а шум как случайный сигнал суммируется по мощности, т.е. увеличивается в $M$ раз.

Таким образом, в работе для случая сверхширокополосных хаотических радиоимпульсов с большой базой получено аналитическое решение для вероятности ошибки обнаружения хаотических радиоимпульсов с помощью детектора огибающей.

Также показано, что использование массива детекторов огибающей позволяет пропорционально увеличить отношение сигнал/шум на приемной стороне. Данный результат является в значительной степени общим, в том смысле, что, хотя он получен для задачи приема сверхширокополосных хаотических радиоимпульсов, природа несущего сигнала становится фактически несущественной после детектирования. Хаотическая природа принимаемого сигнала сказывается на форме функции распределения энергии принятого радиоимпульса и соответственно на оценке вероятности ошибки обнаружения.

Аналогичные выводы относительно увеличения отношения сигнал/шум были ранее сформулированы для систем с накоплением, например, в [15]. Можно также отметить, что такой же принцип увеличения отношения сигнал/шум заложен и в алгоритмы обработки сигнала систем с расширением спектра [16] и т.п.

\section{Финансирование работы}

Работа выполнена при поддержке Российского фонда фундаментальных исследований (грант № 20-02-00877а).

\section{Конфликт интересов}

Авторы заявляют, что у них нет конфликта интересов.

\section{Список литературы}

[1] L. Lu, G.Y. Li, A.L. Swindlehurst, A. Ashikhmin, R. Zhang, IEEE J. Sel. Top. Signal Process., 8 (5), 742 (2014).

[2] R.W. Heath, N. Gonzalez-Prelcic, S. Rangan, W. Roh, A.M. Sayeed, IEEE J. Sel. Top. Signal Process., 10 (3), 436 (2016).

[3] S. Rangan, T.S. Rappaport, E. Erkip, Proc. IEEE, 102 (3), 366 (2014).
[4] L. Jing, E. De Carvalho, P. Popovski, A.O. Martinez, IEEE Trans. Signal Process., 64 (19), 5000 (2016).

[5] K. Thapaliya, Q. Yang, K.S. Kwak, in Int. Conf. on Embedded Software and Systems (ICESS 2007). Lecture Notes in Computer Science (Springer, Berlin-Heidelberg, 2007), vol. 4523, p. 708. DOI: 10.1007/978-3-540-72685-2_65

[6] A.S. Dmitriev, B.E. Kyarginsky, A.I. Panas, S.O. Starkov, Int. J. Bifurcation Chaos, 13 (6), 1495 (2003).

[7] Yu.V. Andreyev, A.S. Dmitriev, E.V. Efremova, A.D. Khilinsky, L.V. Kuzmin, Int. J. Bifurcation Chaos, 15 (11), 3639 (2005).

[8] First Report and Order, FCC 02-48, ET Docket 98-153 (April 2002).

[9] Yu.V. Andreyev, in 2017 Progress in Electromagnetics Research Symposium - Spring (PIERS) (St. Petersburg, 2017), p. 2665. DOI: 10.1109/PIERS.2017.8262203

[10] Yu.V. Andreyev, in 2018 Systems of Signal Synchronization, Generating and Processing in Telecommunications (SYNCHROINFO) (Minsk, 2018), p. 1. DOI: 10.1109/SYNCHROINFO.2018.8457024

[11] Yu. Andreyev, ITM Web Conf., 30, 05029 (2019). DOI: $10.1051 /$ itmconf $/ 20193005029$

[12] D.C. Montgomery, G.C. Runger, Applied statistics and probability for engineers (Wiley, 2014).

[13] A.S. Dmitriev, M. Hasler, A.I. Panas, K.V. Zakharchenko, in Synchronization: theory and application (Springer, Amsterdam, 2003), p. 41-63. DOI: $10.1007 / 978-94-010-0217-2$.

[14] A.S. Dmitriev, M.E. Shirokov, J. Commun. Technol. Electron, 49 (7), 790 (2004).

[15] А.А. Харкевич, Борьба с помехами (Физматлит, М., 1965).

[16] D. Torrieri, Principles of spread-spectrum communication systems (Springer, 2018). DOI: 10.1007/978-3-319-70569-9 